\title{
Aggressive angiomyxoma of the vulva: Case report and review of literature
}

\author{
${ }^{1}$ Dr. Laishram Trinity Meetei, ${ }^{2}$ Dr. Moirangthem Rameshwar Singh, \\ ${ }^{3}$.Dr. Naorem Nabakishore Singh, ${ }^{4}$ Dr. Laishram Deepak Kumar \\ Department of Obstetrics and Gynaecology, \\ *Department of Pathology, Regional Institute of Medical Sciences, Imphal-795004, India
}

\begin{abstract}
Aggressive angiomyxoma (AAM) is a rare mesenchymal benign tumor that preferentially involves the pelvic and the perineal region of relatively young females. The diagnosis of AAM should be considered when a female presents with an atypical vulvoperineal mass. We report a perimenopausal multiparous lady with AAM who presented with a big vulval mass.

Key word : AAM - Aggressive angiomyxoma
\end{abstract}

\section{Introduction :}

It is an extremely uncommon neoplasm of the female pelvis and perineum, first described by Steeper and Rosai in 1983 in nine women [1]. It is locally infiltrative, but non metastasizing lesion mainly involving the vulvar and perineal region of women and girls during the reproductive age group with high frequency of local recurrence. Histologically, it is characterized by a predominantly myxoid stroma and an abundance of thin and thick walled vascular channels. The diagnosis should be considered with any atypical vulvoperineal mass, as incorrect diagnosis may lead to repeated surgical procedures.

\section{Case report :}

A 45 years old multiparous lady presented to the outpatient department with a perineal mass which had increased in size over the last one year causing local discomfort. There were no associated symptoms like pain and no urinary or bowel complaints. Past and menstrual history were unremarkable. On examination, the pedunculated mass $15 \times 13 \times 10 \mathrm{~cm}$ was seen hanging from the right labium majus by a thick pedicle [Fig.1]. It was non tender with surface ulceration. With the differential diagnosis of pedunculated soft tissue tumor, probably lipoma, she was planned for surgical excision. The pedicle was ligated at the base, excised and labial reconstruction was done. On slicing, cut section was homogenous, white fleshy, soft to feel. No areas of haemorrhage or necrosis were identified. The microscopic examination [Fig.2] of the specimen showed tumor composed of scattered spindle shaped and stellate cells, in a loose myxoid stroma which displayed minimal pleomorphism. There were no atypia or increased in mitosis. Many small capillaries and medium sized vessels were seen infiltrating the underlying adipose tissue. The overlying epidermis is focally denued with dense mixed inflammation and vascular proliferation. Features are suggestive of AAM

\section{Discussion :}

Aggressive angiomyxoma is a distinctive, locally infiltrative, mesenchymal neoplasm with a predilection for the pelvis and perineal regions in females. Since its characterization by Steeper and Rosai in 1983, fewer than 150 cases have been reported in the world medical literature, size varying considerably [2]. AAM most commonly occur between $30-40$ years median being 34 years with a range of 16-70 years. Majority are $>10 \mathrm{~cm}$ in the greatest dimension as in our case. The recurrence rate is $36 \%$ on the follow up case 29 patients, one of the largest series [3] and $72 \%$ in other series of 9 patients [1]. Clinical recurrence as late as 14 years have been reported [1] which suggest that the tumor is often very slow growing and long term follow is required before the possibility of recurrence is ruled out. The immunihistochemical evidence of estrogen and progesterone receptors within the neoplastic cells of AAM and its relative site specific location and strong female predominance imply that hormonal factors are active in the evolution of this neoplasm. So, hormonal therapy may have an adjuvant role in the management of selected cases which are not amenable to complete surgical resection [3]. Successful treatment of residual tumor after resection, using gonadotropin releasing hormone $(\mathrm{GnRH})$ agonist has been reported [4].

The differential diagnosis of AAM includes superficial angiomyxoma, angiofibroblastoma, myxoma, myxoid smooth muscle tumors, lipomatous tumors, peripheral nerve sheath tumors, pelvic fibromatosis and low grade myxoid malignant fibrous histiocytoma(myxofibrosarcoma). Clinical diagnosis is usually difficult but with clinical information, histologic diagnosis of AAM can be made without the need for further studies. As 
these tumors have a strong propensity for local recurrence, wide excision without causing morbidity is recommended. Those patients who were managed more conservatively require long term follow up as late recurrence has been reported. Imaging studies with MRI, CT Scan and sonography may be done preoperatively and in the follow of those tumor as extent may be underestimated by physical examination.

\section{References :}

[1]. Steeper TA, Rosai J. Aggressive angiomyxoma of the female pelvis and perineum. Am J Surg Pathol 1983;7:463-75.

[2]. Magtibay PM, Salmon Z, Keeney GL, Podratz KC. Aggrresive angiomyxoma of the female pelvis and perineum : a case series. Int J Gynaecol Cancer 2006;16(1):396-401.

[3]. Fetsch JF, LaskinWB, Lefkowitz M, Kindblom LG, Meis-Kindblom JM. Aggressive angiomyxoma: a clinicopathologic study of 29 female patients. Cancer 1996;78:79-90.

[4]. McCluggage WG,Jameison T,Dobbs SP, Grey A. Aggressive angiomyxoma of the vulva: dramatic response to gonadotropin-releasing hormone agonist therapy. Gynecol Oncol 2006;100: 623-5.



Fig. 1.Mass arising from the vulva.



Fig. 2: Clusters of vessels and stellate cells 